\title{
An Experimental Study on the Optimization of Learners' Cognitive Load in Web-based Learning Environment
}

\author{
Ning Cui ${ }^{1 *}$, Yuan Fang ${ }^{2}$ \\ ${ }^{1}$ Guangdong Nanhua Vocational College of Industry and Commerce, Qingyuan 511510, Guangdong, China \\ ${ }^{2}$ Guangzhou College of Technology and Business, Foshan 528131, Guangdong, China \\ Email: 462677830@qq.com
}

\begin{abstract}
With the global epidemic of COVID-19, online teaching and learning have been carried out on a large scale all over the world, and MOOC has been further developed and applied. Through experiments, this study verified the feasibility of advance organizer strategy, eliminating redundancy strategy and ARCS model design strategy. The results are as follows. (1) In the Web-based learning environment, for the more difficult materials, it is beneficial to reduce the internal cognitive load of learners to provide a certain advance organizer before the learners start formal learning; but for the easier materials, whether or not to provide advance organizer has no significant impact on the learning results of learners. (2) In the Web-based learning environment, it is better to provide advance organizer for the more difficult knowledge by implanting background music into the Web-based learning environment; it can obviously hinder the learners' learning, but it is not obvious when the e-learners learn relatively simple knowledge. (3) In the Web-based learning environment, ARCS model design can stimulate the learning motivation of e-learners and optimize the cognitive load of e-learners.
\end{abstract}

keywords: Web-based learning environment, e-learners, cognitive load, experimental study

\section{Brief introduction and classification of cognitive load}

Cognitive load theory was put forward by Australian psychologist Sweler. J in the 1980s. Based on the theory of limited resources and schema theory, cognitive load theory divides cognitive load into three categories: internal cognitive load, external cognitive load and related cognitive load. The internal cognitive load is caused by the interaction of the difficulty level of learning materials and the learners' original knowledge and experience level (that is, the original schema level); the external cognitive load is determined by the presentation mode of teaching materials and the level of teaching design; the related cognitive load refers to the load that can promote the construction and automation of schema.

\section{Measurement of cognitive load}

Under the network learning environment, whether the learners' cognitive load is optimized and the degree of optimization, the accurate measurement of cognitive load is the key to verify it. Therefore, it is of great significance to design an effective cognitive load measurement method based on the existing research results and the actual situation of this study.

Since Pass vanMerrinboer put forward the structural model of cognitive load in 1994, scholars in various countries have carried out a lot of research on the measurement of cognitive load. So far, the commonly used measurement methods of cognitive load are mainly divided into subjective measurement method, performance measurement method and physiological measurement method.

\subsection{Subjective measurement of cognitive load}

Subjective measurement refers to the assessment of cognitive load based on learners' subjective feelings and experiences in the process of learning, which generally involves the psychological effort, time pressure and task difficulty that learners can perceive. When using subjective measurement, it is usually assumed that learners can accurately express the psychological resources occupied in the learning process, that is to say, learners have the ability to review their cognitive process, and can say the total amount of task difficulty and psychological effort they feel. At present, researchers in various countries have developed a variety of subjective measurement scales for different situations, such as Pass et al's grade 7 and grade 9 cognitive load assessment scale, USAF's scale (SWAT), NASA's TLX scale and Tsang and Velazquez's WP scale gauge. Subjective measurement method can measure learners' cognitive load conveniently and quickly, so it has been widely used.

\subsection{Performance measurement of cognitive load}

Performance measurement refers to the measurement of learners' mental effort by the difficulty of specified learning materials and the learning effect of learners, that is, the measurement of learners' performance in completing a specific task 
to evaluate the cognitive load brought by the task. In general, the higher the complexity of learning materials, the worse the learning effect of learners, that is, the more cognitive load the learning materials bring to learners. The measurement indexes of performance measurement method include: task reaction time, sub task reaction time, task accuracy, learning speed, learning error rate, etc.

\subsection{Physiological measurement of cognitive load}

Physiological measurement assumes that learners have various physiological responses to cognitive load in the process of learning or completing tasks, such as heart activity, eye movement and brain wave amplitude. Physiological measurement can well reflect the detailed trend and model of cognitive load, such as instantaneous load, average load, cumulative load and peak load, but at the same time, physiological measurement needs certain professional instruments as assistance, which has certain limitations in the process of application. Therefore, physiological measurement is generally not used in practice when there is no very high requirement for measuring cognitive load.

\subsection{Measurement methods used in this study}

After considering the advantages and limitations of various measurement methods and combining with the actual situation, we decided to adopt the following measurement methods.

\subsubsection{Subjective measurement}

After the completion of the learning task, the cognitive load generated in the learning process was measured subjectively. The mental effort and task difficulty of the learners were measured by the rating scale. The nine level rating scale of cognitive load designed by Pass et al.

\subsubsection{Performance measurement}

The level of cognitive load produced by learners in the learning process is directly reflected in the learning results, so the measurement of learners' memory and transfer performance can indirectly measure the level of cognitive load in the learning process. Performance measurement is carried out through the test questionnaire.

\section{Experimental study on the optimization of cognitive load of e-learners}

\subsection{To provide an experiment for the advance organizer to optimize the internal cognitive load of e-learners}

In web-based learning environment, learners' internal cognitive load is mainly determined by learners' original knowledge level and material difficulty. Therefore, this experiment assumes that by providing learners with prior knowledge, learners will have a certain foundation of schema construction in the knowledge field they are about to learn. In this way, learners' internal cognitive load will remain at a low level even when they are learning more difficult materials. Of course, if the difficulty of learning materials is small, no matter whether the learners have prior knowledge or not, their internal cognitive load will remain at a low level When learning, cognitive load is at a low level. Based on this hypothesis, this study provides advance organizers to optimize the internal cognitive load of e-learners, so as to explore the influence of advance organizers on the internal cognitive load of learners in the web-based learning environment and the optimization effect.

\subsubsection{Experimental design}

In order to avoid the learning fatigue effect of the subjects, this experiment adopts the intersubject experiment design of 2 (advance organizer) $\times 2$ (material difficulty). The experiment is divided into two groups, each time is divided into two groups. The first time, the two groups only learn the more difficult learning materials, the second time, the other two groups only learn the less difficult learning materials. The experiment is divided into four groups: A, B, C and D A and C were the experimental groups, B and D were the control groups. The advance organizer would present the materials before the formal learning materials of the experimental group in each experiment, explain the basic knowledge and professional terms involved in the materials or give feedback to the subjects in other ways, while the control group would learn the materials directly. The dependent variables were mental effort, difficulty evaluation of materials, and memory and transfer test scores.

\subsubsection{Experimental test}

The items to be tested in this experiment are: Learners' prior knowledge examination, cognitive load measurement in the learning process, and learners' memory and transfer test scores. Previous knowledge check mainly investigates the correlation between learners' original knowledge and experience and the materials to be learned, mainly through the test questionnaire; after learning, learners' cognitive load can not be directly measured, but can be measured through subjective 
assessment and physiology. Due to the limitation of experimental conditions, this study only uses pass's nine level selfassessment scale.In addition, the test of memory and transfer performance is also an effective way to indirectly measure the cognitive load of learners in the learning process.

\subsubsection{Experimental materials}

The experimental materials involved in this study are processed by premiere CC and DW CC. The most difficult materials are the optical characteristics of cameras, and the easier materials are the proximity principle in interpersonal communication. The materials are mainly presented to learners in the form of videos, and the videos are put under the corresponding links of learning web pages. The optical characteristics of the camera is about the physical relationship among the focal length, relative aperture and field angle of view of the camera lens. The playback time of the learning material is 307 seconds. The principle of proximity in interpersonal communication tells that in the process of interpersonal communication, people are more likely to have a good impression on the people they are familiar with or know. The material also explains the single exposure effect in psychology. The playback time of the video material is 402 seconds. The first organizer is added after the two videos are processed by PRCC. The first organizer of camera optical characteristics is the descriptive explanation of focal length, relative aperture and field angle. For example, the description of focal length is the distance from the lens center to the image plane such as the base plate or CCD, which can be shown in the figure below (Figure 1). The first organizer is also processed by PRCC.

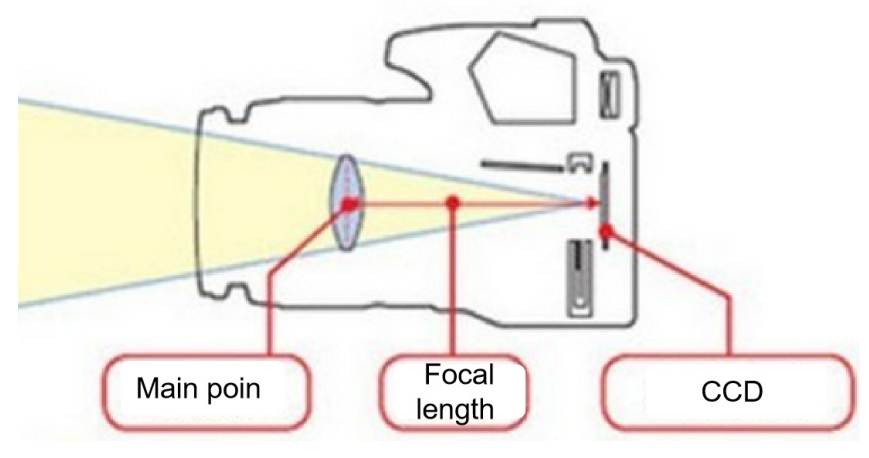

Figure 1. material of advance organizer

\subsubsection{Experimental object}

A total of 53 subjects from Guangdong Nanhua Vocational College of industry and Commerce (China) participated in this experiment, including 28 boys and 25 girls, aged between 18 and 25 years old, with an average age of 21.3 years old and a college degree. The subjects were randomly assigned to group A, B, C and D, including 13 in group A, 13 in group B, 13 in group C and 14 in Group D. The experiment is divided into two times, both in the computer room, each subject has a computer, a pair of headphones, the first experiment for group A, B, learning materials for the optical characteristics of the camera. Firstly, the original knowledge and experience of the two groups were investigated, and then they took a rest of three minutes; secondly, the knowledge of camera optical characteristics was learned. In group A, the video materials of the advance organizer were added, while in group B, the video materials without the advance organizer were learned, and the total learning time was 10 minutes; finally, the cognitive load, memory and transfer performance of the learners were measured. The process of the second experiment is the same as that of the first one, except that the subjects are changed into groups $\mathrm{C}$ and $\mathrm{D}$, and the learning material is the principle of proximity in interpersonal communication. After each experiment, each subject was given a small gift.

\subsubsection{Collection, analysis and processing of experimental data}

(1) Test of prior knowledge, short term memory and information search ability

The main content of this test is the learners' previous knowledge and short-term memory level, but the information technology level involved is the possible interference factor of the learners in this experiment. In order to exclude the situation that the learners will not use the computer, each learner will be given a prompt to help them quickly search the target during the test, and the information search of the two groups of subjects will be tested after the prompt Whether there is a significant difference in ability.

Firstly, the previous knowledge, short-term memory capacity and information technology level of group A and B were tested by questionnaire, and the descriptive results are shown in Table 1. 
Table 1. Previous knowledge, short-term memory and information searching level of learners in A\&B groups

\begin{tabular}{cccc}
\hline Test content & Material type & Group a learners & Group B learners \\
\hline Previous knowledge level & Hard material & $5.16 \pm 0.35$ & $5.02 \pm 0.28$ \\
Short term memory level & Digital memory & $6.25 \pm 0.50$ & $6.11 \pm 0.75$ \\
The level of information technology involved & Information search time & $8.77 \pm 0.5 \mathrm{~s}$ & $8.69 \pm 0.6 \mathrm{~s}$ \\
\hline
\end{tabular}

Then, the test results of learners' prior knowledge, short-term memory and information technology level were analyzed by one-way ANOVA, and the results are shown in Table 2.

Table 2. Variance analysis of previous knowledge, short-term memory and information searching level of learners in A\&B groups

\begin{tabular}{ccc}
\hline Test type & F value & Significance \\
\hline Prior knowledge & 0.072 & 0.791 \\
Immediate memory span & 0.016 & 0.901 \\
Information search level & 0.007 & 0.935 \\
\hline
\end{tabular}

According to the one-way ANOVA of the two groups of learners, the information technology level factor $\mathrm{f}(0.007)$ $=0.935$, the significance is greater than 0.05 , which indicates that there is no significant difference in the information technology level between the two groups of learners in this experiment, and the interference factors of the information search ability difference in this experiment can be excluded. Similarly, it can be seen from table 2 that the significance levels of learners' prior knowledge and short-term memory capacity are $\mathrm{f}(0.072)=0.791$ and $\mathrm{f}(0.016)=0.901$, respectively, and the significance is greater than 0.05 , indicating that there is no significant difference between the two groups in terms of prior knowledge and short-term memory capacity.

Secondly, in the process of testing the prior knowledge, short-term memory level and information search ability of the $\mathrm{C}$ and $\mathrm{D}$ groups, it is found that there are significant differences in the easier material learning between the two groups at three levels: $\mathrm{F}(0.066)=0.799>0.05, \mathrm{f}(0.015)=0.902>0.05, \mathrm{f}(0.005)=0.945>0.05$, and the significant difference is not obvious.

(2) Cognitive load, memory and transfer performance of learners

After the end of learning, the experimental data of the experimental group and the control group about the difficulty evaluation of learning materials, the degree of psychological effort in the learning process, and the memory and transfer performance after learning were collected. The results of the experimental data are shown in Table 3.

Table 3. Cognitive load and performance of learners in the four groups

\begin{tabular}{ccccc}
\hline & \multicolumn{3}{c}{ Group } \\
\cline { 2 - 5 } Test content & $\mathrm{A}$ & $\mathrm{B}$ & $\mathrm{C}$ & $\mathrm{D}$ \\
\hline Difficulty evaluation & 5.67 & 6.69 & 3.58 & 3.54 \\
Mental effort & 5.82 & 6.74 & 3.86 & 3.81 \\
Memory and transfer performance & 6.82 & 5.75 & 7.42 & 7.50 \\
\hline
\end{tabular}

First of all, the ANOVA analysis of the experimental data of group A and group B shows that the significant differences in the difficulty evaluation of materials, the mental efforts of learners and the scores of memory and transfer after learning between group A and group B are $\mathrm{f}(8.780)=0.007, \mathrm{f}(6.584)=1.017, \mathrm{f}(4.370)=0.047, \mathrm{P}<0.05$ There were significant differences between experimental group A and control group B at three levels. Specific ANOVA data are shown in Table 4.

Table 4. Significant variance analysis of the learning results of learners in A\&B groups on the three levels

\begin{tabular}{ccc}
\hline Analysis content & F value & Significance \\
\hline Prior knowledge & 8.780 & 0.007 \\
Immediate memory span & 4.370 & 0.047 \\
Information search level & 6.584 & 0.017 \\
\hline
\end{tabular}


Similarly, further ANOVA analysis on the data of learning results of group C and group D showed that the significant differences in difficulty evaluation, mental effort, memory and transfer performance were $\mathrm{f}(0.034)=0.855, \mathrm{f}(0.041)=$ $0.840, \mathrm{f}(0.027)=0.870$ respectively, and the significant results were all greater than 0.05 . The specific data are shown in Table 5, which shows that it is feasible to use the easier learning materials There was no significant difference between the experimental group and the control group.

Table 5. Significant variance analysis of the learning results of learners in C\&D groups on the three levels

\begin{tabular}{ccc}
\hline Analysis content & F value & Significance \\
\hline Prior knowledge & 0.034 & 0.855 \\
Immediate memory span & 0.041 & 0.840 \\
Information search level & 0.027 & 0.870 \\
\hline
\end{tabular}

\subsubsection{Discussion on the experimental results}

Through this experiment, we can draw the following conclusions: for the learning of more difficult materials in the network learning environment, providing a certain advance organizer before the learners start formal learning is conducive to reducing the cognitive load, which is conducive to the learners' learning, and has a promoting effect on the improvement of learners' learning performance; but for the easier learning materials, providing advance organizer and advance organizer can improve the students' learning performance Whether or not has no significant effect on learners' learning results, and even has a certain hindrance in academic performance. This experiment further verifies the experimental hypothesis. In addition, the students in this experiment also made the corresponding difficulty evaluation on the two kinds of learning materials. From the measurement results, the difficulty coefficient of the optical principle of the camera is far greater than the proximity principle in interpersonal communication, which indicates that the experimental materials selected in this experiment meet the expected standard. Finally, this experiment verifies the strategy of providing advance organizers to optimize the internal cognitive load of e-learners, and provides experimental data support for it.

\subsection{An experiment on the influence of background music on learners' cognitive load in web-based learning environment}

The output of redundant information is one of the influencing factors of learners' external cognitive load in e-learning environment. This experiment assumes that the occurrence of redundant information unrelated to effective learning resources in e-learning environment will hinder learners' learning and aggravate the external cognitive load of e-learners, especially when the knowledge they want to learn is more difficult In order to be significant, this experiment chooses to implant background music that learners can't turn off in the network learning environment to verify the hypothesis.

\subsubsection{Experimental design}

This experiment adopts a mixed design of 2 (background music: Yes, no) $\times 2$ (material difficulty: difficult, easy). The former is the inter subject factor, while the latter is the intra subject factor. The background music is directly implanted into the teaching system. The experiment is divided into two groups: Group a and group B. in group A, the background music is implanted into the more difficult learning materials and the less difficult learning materials, The dependent variables in the experiment were learners' difficulty evaluation of materials and their scores of memory and transfer.

\subsubsection{Experimental test and experimental materials}

The test method used in this experiment is the same as experiment 1 . The test content also includes three aspects: Learners' prior knowledge check, cognitive load measurement in the learning process, learners' memory and transfer test scores. In the selection of experimental materials, the more difficult experimental materials still choose the optical principle of the camera, and the more difficult materials choose the proximity principle in interpersonal communication. However, the treatment of experimental materials is different from Experiment 1. In this experiment, in addition to PR CC, dream weaver $\mathrm{CC}$ is also used to implant background music.

\subsubsection{Experimental process}

A total of 28 subjects from Guangdong Nanhua Vocational College of industry and commerce were selected in this experiment, including 16 boys and 12 girls, aged between 18 and 24 years old, with an average age of 21.8 years old. The subjects were randomly assigned to group A and group B, 14 in group A and 14 in group B. Group A is the experimental group, that is, background music will be implanted in the whole process of learning, while group B is the control group, there will be no background music in the whole process of learning. The experiment was carried out in the computer room, with each subject having a computer and a pair of earphones. First, the original knowledge and experience of the two groups were 
investigated, and then they had a rest of three minutes. Secondly, both groups A and B learned the optical characteristics of cameras at the same time. After learning, they continued to learn the proximity principle in interpersonal communication, and the total learning time of each difficult material All of them took 10 minutes (including pause and thinking time in the process of playing video); finally, the cognitive load and memory and transfer performance of the learners were measured. After the experiment, each subject was given a small gift.

\subsubsection{Data statistics}

(1) Prior knowledge test

By examining the previous knowledge and short-term memory capacity of group A and B, the descriptive results are shown in Table 6.

Table 6. Previous knowledge level and short-term memory capacity of learners in A\&B groups

\begin{tabular}{cccc}
\hline Test content & Material type & Group A learners & Group B learners \\
\hline \multirow{2}{*}{ Previous knowledge level } & Hard material & $5.18 \pm 0.2$ & $5.00 \pm 0.2$ \\
& Easy material & $5.31 \pm 0.2$ & $5.45 \pm 0.2$ \\
Immediate memory span & Digital memory & $6.35 \pm 0.5$ & $6.57 \pm 0.5$ \\
\hline
\end{tabular}

One way ANOVA was used to analyze the previous knowledge level and short-term memory capacity of group A and group B. The results showed that the results of one-way ANOVA on the more difficult materials were $f(0.05)=0.945$, and the results of one-way ANOVA on the easier materials were $\mathrm{f}(0.011)=0.917$, The results of one-way ANOVA on short-term memory capacity were $\mathrm{f}(0.122)=0.730$, the significance of the three results were greater than 0.05 , indicating that there was no significant difference in the previous knowledge level and short-term memory capacity between the two groups.

(2) Cognitive load measurement

After the experiment, the descriptive results of data collection and analysis of the two groups of learners are shown in Table 7.

Table 7. Descriptive result of the statistics of two groups

\begin{tabular}{cccccc}
\hline Test type & & $\mathrm{N}$ & Mean value & Minimum value & Maximum value \\
\hline $\begin{array}{c}\text { Difficulty evaluation of difficult } \\
\text { materials }\end{array}$ & $\mathrm{A}$ & 14 & 7.4929 & 5.50 & 9.00 \\
& $\mathrm{~B}$ & 14 & 6.6786 & 4.30 & 8.40 \\
$\begin{array}{c}\text { Difficulty evaluation of easy } \\
\text { materials }\end{array}$ & $\mathrm{A}$ & 14 & 3.6786 & 2.40 & 4.60 \\
& $\mathrm{~B}$ & 14 & 3.6357 & 2.40 & 4.60 \\
Difficult material achievement & $\mathrm{A}$ & 14 & 5.1751 & 2.80 & 7.00 \\
& $\mathrm{~B}$ & 14 & 6.1000 & 4.60 & 7.70 \\
Easy material achievements & $\mathrm{A}$ & 14 & 7.3571 & 5.00 & 9.50 \\
\hline
\end{tabular}

One way ANOVA was used to analyze the difficulty evaluation of learning materials and the memory and transfer performance of group A and group B. the significant results were as follows: in the learning of more difficult materials, the significance of difficulty evaluation of materials was $\mathrm{f}(4.402)=0.046$, and the significance of memory and transfer performance was $\mathrm{f}(5.355)=0.029$; In the learning of easier materials, the significance of difficulty evaluation, memory and transfer scores of the two groups were $\mathrm{f}(0.036)=0.852, \mathrm{f}(0.113)=0.740$, respectively.

\subsubsection{Discussion}

From the significance analysis of the two groups of data, it can be seen that in the learning of more difficult knowledge, by implanting background music into the network learning environment, it has an obvious hindrance to the learners' learning, but this hindrance is not obvious when the network learners learn more simple knowledge. The experimental results confirm the hypothesis proposed in this study to a certain extent, but there is a certain gap between them. After analysis, on the one hand, when learners are learning relatively simple knowledge, their cognitive resources are still free to some extent, and even adding background music unrelated to the learning topic is not enough to interfere. For some learners, it may even become a factor to increase the relevant cognitive load; on the other hand, it can be used for reference It is not obvious to add 
background sound only, but it will be more obvious if the advertising window can be implanted at the same time.

\subsection{An experiment of ARCS model design to optimize the related cognitive load of e-learners}

When learners are learning, they can use the remaining cognitive resources for information processing directly related to effective learning resources without cognitive overload. Through these processing, the knowledge involved in learning resources will be integrated into the existing knowledge structure of learners to promote the construction of learners' schema, although these processing will also cause learners' cognitive load However, the increase of these loads is directly related to the knowledge goals to be achieved, which can directly lead to the improvement of learners' academic performance. Such loads are called associative cognitive load or effective cognitive load. But not in all cases, learners will actively increase their relevance cognitive load (such as active knowledge reorganization, comparison, reasoning, etc.), the increase of relevance cognitive load has a great relationship with learners' initiative and enthusiasm. Therefore, improving learners' interest in learning is conducive to increasing learners' relevance cognitive load.

Based on the above considerations, the study assumes that the ARCS model can be used in the design of web-based learning environment (mainly through the use of official account platform of WeChat, which releases knowledge points related to curriculum contents, related photographic works and corresponding encouraging words, and establishes WeChat group and QQ group to increase interaction among organizers, organizers and subjects; and tells the experimental group to be tested. At the end of the course, the students with excellent results will be organized to take on-the-spot photos; the subjects in the experimental group will be informed that they will issue certificates to the students with excellent results after the end of the course.) To enhance the interest and motivation of e-learners can increase the cognitive load of relevance and promote the improvement of learning performance.

\subsubsection{Dxperimental design}

This experiment adopts 1 (with ARCS Model Design) $\times 1$ (without ARCS Model Design) comparative experiment design between subjects. The learning materials are selected from "random talk on the practicability and artistry of photography" on the e-learning platform of love course. The course is divided into six explanation units, which mainly describes the basic principles of photography, basic skills of photography, practicality and artistry of photography, and experimental organization They were divided into group A and group B. group A was the experimental group and group B was the control group. The experimental group was accompanied with ARCS model design in the whole learning process to promote the improvement of learning interest, while the control group was only assigned learning tasks, and there was no ARCS model design as an auxiliary in the whole learning process. Memory and transfer performance of two groups after dependent variable learning.

\subsubsection{Experimental test}

This experiment is a tracking service experiment. The course has six explanation units, which need a week's learning time for online learners. In order to give the two groups a real online learning environment, they will disturb the two groups as little as possible in a week's learning time. Therefore, this experiment will no longer test the working memory capacity and the cognitive load of each lecture of the two groups. The content of this experiment mainly includes the previous knowledge check of the two groups and the memory and transfer performance after learning. Through the memory and transfer performance, we can infer the interest level of the two groups in the whole learning process, and then understand arcs The role of model design in optimizing the relevant cognitive load of e-learners.

\subsubsection{Experimental process}

A total of 93 students, including 53 boys and 40 girls, aged between 18 and 25 years old, with an average age of 21.3 years old, were selected from multimedia class 1 and class 2 of Guangdong Nanhua Vocational College of industry and commerce. Multimedia class 1 is named group A, and multimedia class 2 is named group B. There are 48 students in group A and 45 students in group B. Group A is the subject group, that is, the subjects in the whole process of learning will have ARCS model aided design, group B is the control group, the subjects in the whole process of learning will not have ARCS model aided design. There are 7 students, including 4 in group A and 3 in group B. the seven students will be allocated 7 computers for one week in the 308 laboratory room of the experimental building of Guangdong Nanhua Vocational College of industry and commerce. The experiment lasted for one week. The learners chose the time period and controlled the learning progress by themselves. However, both groups were informed of the learning task and the time to check the learning results. Before learning, the two groups were checked for prior knowledge, and then the data were counted. After the whole learning process, the memory and transfer performance of the two groups were measured.

\subsubsection{Experimental data analysis}

(1) Prior knowledge check

First of all, through the prior knowledge examination of a and B groups, the descriptive results are shown in Table 8 . 
Table 8. Descriptive result of the examination of previous knowledge of Ss of A\&B groups

\begin{tabular}{ccccc}
\hline Group & N & Mean value & Minimum value & Maximum value \\
\hline A & 48 & 6.0135 & 2.72 & 9.06 \\
B & 45 & 6.1120 & 1.72 & 9.38 \\
\hline
\end{tabular}

Secondly, the one-way ANOVA was used to analyze the level of prior knowledge of group A and group B. The results showed that the result of one-way ANOVA of prior knowledge of group A and group B was $\mathrm{f}(0.105)=0.745>0.05$, indicating that there was no significant difference in the level of prior knowledge of group A and group B.

(2) Cognitive load measurement

After the experiment, the descriptive results of data collection and analysis of the two groups are shown in Table 9.

Table 9. Descriptive result of the performance of memory and transfer of learners in A\&B groups

\begin{tabular}{ccccc}
\hline Group & N & Mean value & Minimum value & Maximum value \\
\hline A & 48 & 6.960 & 2.3 & 9.0 \\
B & 45 & 5.940 & 2.6 & 9.3 \\
\hline
\end{tabular}

One way ANOVA was used to analyze the memory and transfer performance of group A and group B, and the significant result was $\mathrm{f}(18.854)=0.000<0.05$, which indicated that there were significant differences in memory and transfer performance between the two groups. The specific results are shown in table 10.

Table 10. Variance analysis result of the performance of memory and transfer of learners in A\&B groups

\begin{tabular}{cccccc}
\hline & Sum of squares & df & Mean square & F & Significance \\
\hline Between groups & 24.184 & 1 & 24.184 & 18.854 & 0.000 \\
\hline
\end{tabular}

\subsubsection{Experimental discussion}

This experiment is based on the ARCS model proposed by the famous American instructional design expert Keller, and combines the characteristics of the current network learning environment. It designs the WeChat official account platform, WeChat group, QQ group, certificates for outstanding learners, and organizing the experimental group participants to shoot in the field to stimulate the learning motivation of the weblearners and improve their learning interest. And optimize the cognitive load of e-learners. According to the experimental results, the relevant data of this experiment verify the hypothesis, and prove that the design of ARCS Model in e-learning environment is conducive to optimize the relevant cognitive load of e-learners. However, due to the time relationship, the investigation period of this experiment is only one week. In the followup research, we need to further increase the investigation period, expand the scope of investigation objects and increase the types of courses, so as to further demonstrate the universality of the cognitive load related to the optimization of online learning by ARCS model design.

\section{Summary and prospect}

This study verified through experiments. (1) The organizer optimizes the internal cognitive load of e-learners. (2) The interference of irrelevant information (inserting background music) hinders the external cognitive load of e-learners. (3) The design of ARCS model optimizes the related cognitive load of e-learners. Hope to be able to provide some inspiration for network learners and the construction of network learning environment in the future. The influencing factors and optimization strategies of learners' cognitive load in the e-learning environment mentioned in the study need further empirical research and more practical tests in the later stage, and collect data from more experimental studies to further verify the advantages and disadvantages of the optimization strategies and models of learners' cognitive load in the e-learning environment, and then improve and promote them.

\section{References}

[1] Chongyong Sun. Measurement of cognitive load and its application in multimedia learning. Higher education research. 2015; (12): 86-86. 
[2] Qimei An,Hong Wu. Review of cognitive load theory. Research progress in psychology. 2015; (5): 50-55.

[3] Yuan Gao, Zhenzhen Huang,Jihong Li, Ronghuai Huang. Cognitive load in intelligent learning environment. Open education research. 2017; 23(01): 56-64.

[4] Jing Li, Shulan Yu, Dong Jin. design and knowledge presentation of balanced cognitive load. Research on audio visual education. 2018; (03): 1-5.

[5] Dunleavy M., Dede C. (2014) Augmented Reality Teaching and Learning. In: Spector J., Merrill M., Elen J., Bishop M. (eds) Handbook of Research on Educational Communications and Technology. Springer, New York, NY. https://doi. org/10.1007/978-1-4614-3185-5 59.

[6] Devos Hannes, Gustafson Kathleen, Ahmadnezhad Pedram, et al. Psychometric Properties of NASA-TLX and Index of Cognitive Activity as Measures of Cognitive Workload in Older Adults. Brain Sci. 2020; 10(12): 994. 